\title{
Discrete Time Stability Analysis of a Three Species Eco System
}

\author{
D.Ravi kiran ${ }^{1, *}$, B.Rami reddy ${ }^{2}$, N.Ch.Pattabhiramacharyulu ${ }^{3}$ \\ ${ }^{1}$ Department of Basic Science, Shri Vishnu Engineering College for Women, Bhimavaram 534202, India \\ ${ }^{2}$ Department of Mathematics, Hindu College, Guntur 522003, India \\ ${ }^{3}$ Former faculty, Department of mathematics, National Institute of Technology, Warangal 506002, India \\ *Corresponding Author: duvvuriravikiran9@gmail.com
}

Copyright $@ 2014$ Horizon Research Publishing All rights reserved.

\begin{abstract}
In this paper, a three species eco system, involving three pairs is considered to examine the local asymptotic stability: A Prey-Predator, a Commensal-Host and an Ammensal-Enemy. Among the three species, one plays a dual role: A host and an enemy. Time is considered as a discrete unit and the system is modeled as a set of three difference equations. All the equilibrium states are identified and the local asymptotic stability of some of the equilibrium states is examined by considering the perturbation equations. It is observed that among the states, the state in which the Prey and its Host species are washed out(extinct), is spectrally stable and the state where the Predator/Ammensal species is washed out, is asymptotically stable. The results are illustrated with two dimensional plots as well as surface plots.
\end{abstract}

Keywords Prey, Predator, Ammensal, Commensal, Enemy, Host, Discrete Time, Asymptotic

\section{Introduction}

Prey-Predator ecological system was presented by Lotka [1] and Volterra [2] in their classical model. Inspired by this model, several researchers made significant contributions in this area by considering various special types of interactions between the prey and the predator. This has been the motivation for others in bringing a third species into the system thus forming a three species ecological system. More information with examples of all ecological interactions can be found in the book by Paul colinvaux[7]. Recently, Seshagiri rao and Pattabhiramacharyulu[15] worked on three species system by considering interactions like Prey-Predator, Commensal - Host, between the three species, which motivated the present authors[16] to consider a three species Eco system with species $\mathrm{S}_{1}, \mathrm{~S}_{2}$ and $\mathrm{S}_{3}$ simultaneously having the interactions of Prey-predation, commensalism and ammensalism, as shown in Figure 1, with time as a continuous unit. $S_{1}$ and $S_{2}$ form a Prey-Predator pair. That is,
$S_{2}$ depends on $S_{1}$ for its survival. $S_{1}$ and $S_{3}$ form a Commensal - Host pair. That is, $S_{3}$ acts as host to $S_{1}$ without itself being affected. And $\mathrm{S}_{2}$ and $\mathrm{S}_{3}$ form an Ammensal Enemy pair. That is, $S_{3}$ inhibits $S_{2}$ without itself being affected. Further the present authors [17] have also carried out a numerical analysis of the same. In the present paper, the three species Ecosystem with time as discrete unit is considered. The equilibrium states are identified and the asymptotic stability of the equilibrium states is examined. A few of them are presented here.

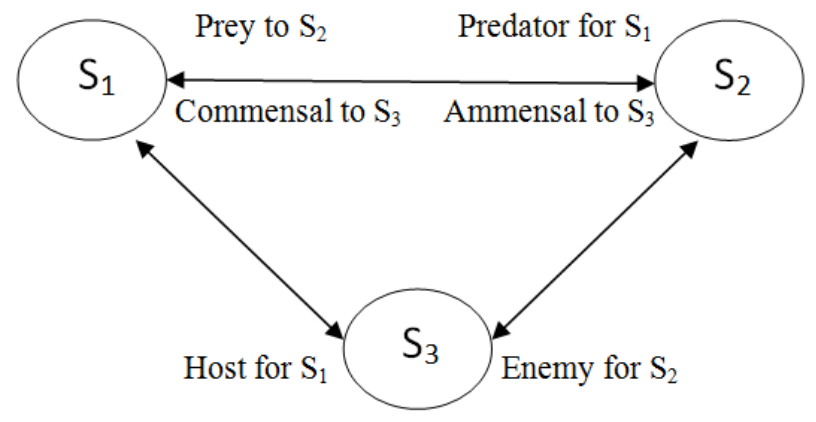

Figure 1. A 3 species ecosystem

\section{Notation}

$\mathrm{N}_{\mathrm{i}}$ : The population of $\mathrm{S}_{\mathrm{i}}, \mathrm{i}=1,2,3$.

$a_{i}$ : The Natural growth rate of $S_{i}, i=1,2,3$.

$a_{i i}$ : Self inhibition coefficient of $S_{i}, i=1,2,3$. (The rate of decrease of $\mathrm{N}_{\mathrm{i}}$ due to insufficient natural resources of $\mathrm{S}_{\mathrm{i}}$ )

$a_{12}$ : The rate of decrease of $S_{1}$ due to inhibition by $S_{2}$.

$a_{13}$ : The rate of increase of the $S_{1}$ due to the promotion by its host $\mathrm{S}_{3}$.

$a_{21}$ : The rate of increase of the $S_{2}$ due to its attacks on $S_{1}$

$a_{23}$ : The rate of decrease of the $S_{2}$ due to the harm caused by its enemy $\mathrm{S}_{3}$.

$\mathrm{p}\left(=\mathrm{a}_{12} / \mathrm{a}_{11}\right)$ : Coefficient of Prey /Commensal inhibition of 
the predator.

$\mathrm{q}\left(=\mathrm{a}_{13} / \mathrm{a}_{11}\right)$ : Coefficient of Commensalism.

$\mathrm{r}\left(=\mathrm{a}_{21} / \mathrm{a}_{22}\right)$ : Coefficient of predator consumption of the prey.

$\mathrm{S}\left(=\mathrm{a}_{23} / \mathrm{a}_{22}\right)$ : Coefficient of Ammensalism.

\section{The Model Equations}

Based on the interactions of the species $S_{1}, S_{2}$ and $S_{3}$, the model equations respectively are

$$
\begin{gathered}
\frac{d N_{1}}{d t}=a_{1} N_{1}-a_{11} N_{1}^{2}+a_{13} N_{1} N_{3}-a_{12} N_{1} N_{2} \\
\frac{d N_{2}}{d t}=a_{2} N_{2}-a_{22} N_{2}^{2}+a_{21} N_{1} N_{2}-a_{23} N_{2} N_{3} \\
\frac{d N_{3}}{d t}=a_{3} N_{3}-a_{33} N_{3}^{2}
\end{gathered}
$$

Considering time as discrete unit, we have from the model equations

$$
\begin{aligned}
& \mathrm{N}_{1}(\mathrm{t}+1)=\alpha_{1} \mathrm{~N}_{1}(\mathrm{t})\left[\begin{array}{l}
1-\frac{\mathrm{a}_{11}}{\alpha_{1}} \mathrm{~N}_{1}(\mathrm{t})-\frac{\mathrm{a}_{12}}{\alpha_{1}} \mathrm{~N}_{2}(\mathrm{t}) \\
+\frac{\mathrm{a}_{13} \mathrm{~N}_{3}(\mathrm{t})}{\alpha_{1}}
\end{array}\right] \\
& \mathrm{N}_{2}(\mathrm{t}+1)=\alpha_{2} \mathrm{~N}_{2}(\mathrm{t})\left[1-\frac{\mathrm{a}_{22}}{\alpha_{2}} \mathrm{~N}_{2}(\mathrm{t})+\frac{\mathrm{a}_{21}}{\alpha_{2}} \mathrm{~N}_{1}(\mathrm{t})-\frac{\mathrm{a}_{23}}{\alpha_{2}} \mathrm{~N}_{3}(\mathrm{t})\right] \\
& \mathrm{N}_{3}(\mathrm{t}+1)=\alpha_{3} \mathrm{~N}_{3}(\mathrm{t})\left[1-\frac{\mathrm{a}_{33}}{\alpha_{3}} \mathrm{~N}_{3}(\mathrm{t})\right]
\end{aligned}
$$

where $1+\mathrm{a}_{1}=\alpha_{1}, 1+\mathrm{a}_{2}=\alpha_{2}$ and $1+\mathrm{a}_{3}=\alpha_{3}$.

Assuming the notation

$\mathrm{K}_{1}=\frac{\mathrm{a}_{11}}{\alpha_{1}}, \mathrm{~K}_{2}=\frac{\mathrm{a}_{22}}{\alpha_{2}}, \mathrm{~K}_{3}=\frac{\mathrm{a}_{33}}{\alpha_{3}}, \beta_{12}=\frac{\mathrm{a}_{12}}{\alpha_{1}}$,

$\beta_{21}=\frac{a_{21}}{\alpha_{2}}, \gamma_{13}=\frac{a_{13}}{\alpha_{1}}, \gamma_{23}=\frac{a_{23}}{\alpha_{2}}$,

the equations (3.1) become

$$
\left.\begin{array}{l}
N_{1}(t+1)=\alpha_{1} N_{1}(t)\left[1-K_{1} N_{1}(t)-\beta_{12} N_{2}(t)+\gamma_{13} N_{3}(t)\right] \\
N_{2}(t+1)=\alpha_{2} N_{2}(t)\left[1-K_{2} N_{2}(t)+\beta_{21} N_{1}(t)-\gamma_{23} N_{3}(t)\right. \\
N_{3}(t+1)=\alpha_{3} N_{3}(t)\left[1-K_{3} N_{3}(t)\right]
\end{array}\right\}
$$

The Equilibrium States of the system are obtained by considering

$$
\mathrm{N}_{\mathrm{i}}(\mathrm{t}+1)=\mathrm{N}_{\mathrm{i}}(\mathrm{t}), \quad \mathrm{i}=1,2,3 .
$$

Solving (4.1), we get eight equilibrium states which can be spread over four distinct classes given by

Fully washed out state:

$$
\mathrm{E}_{1}: \overline{\mathrm{N}}_{1}=0, \overline{\mathrm{N}}_{2}=0, \overline{\mathrm{N}}_{3}=0 .
$$

States in which two species are washed out:

$$
\begin{aligned}
& \mathrm{E}_{2}: \overline{\mathrm{N}}_{1}=0, \overline{\mathrm{N}}_{2}=0, \overline{\mathrm{N}}_{3}=\frac{1}{\mathrm{~K}_{3}}\left(1-\frac{1}{\alpha_{3}}\right) \\
& \mathrm{E}_{3}: \overline{\mathrm{N}}_{1}=0, \overline{\mathrm{N}}_{2}=\frac{1}{\mathrm{~K}_{2}}\left(1-\frac{1}{\alpha_{2}}\right), \overline{\mathrm{N}}_{3}=0 \\
& \mathrm{E}_{4}: \overline{\mathrm{N}}_{1}=\frac{1}{\mathrm{~K}_{1}}\left(1-\frac{1}{\alpha_{1}}\right), \overline{\mathrm{N}}_{2}=0, \overline{\mathrm{N}}_{3}=0
\end{aligned}
$$

States in which only one species is washed out :

$$
\begin{aligned}
\mathrm{E}_{5}: \overline{\mathrm{N}}_{1} & =0, \\
\overline{\mathrm{N}}_{2} & =\frac{1}{\mathrm{~K}_{2}}\left(1-\frac{1}{\alpha_{2}}-\frac{\gamma_{23}}{\mathrm{n}_{3}}\left(1-\frac{1}{\alpha_{2}}\right)\right), \\
\overline{\mathrm{N}}_{3} & =\frac{1}{\mathrm{~K}_{3}}\left(1-\frac{1}{\alpha_{3}}\right) \\
\mathrm{E}_{6}: & \overline{\mathrm{N}}_{1}=\frac{1}{\mathrm{~K}_{1}}\left(1-\frac{1}{\alpha_{2}}-\frac{\gamma_{13}}{\mathrm{n}_{3}}\left(1-\frac{1}{\gamma_{3}}\right)\right), \\
\overline{\mathrm{N}}_{2} & =0, \\
\overline{\mathrm{N}}_{3} & =\frac{1}{\mathrm{~K}_{3}}\left(1-\frac{1}{\alpha_{3}}\right)
\end{aligned}
$$

$\mathrm{E}_{7}$ :

$$
\begin{aligned}
& \overline{\mathrm{N}}_{1}=\frac{\beta_{12}\left(\frac{1}{\alpha_{2}}-1\right)-\mathrm{K}_{2}\left(\frac{1}{\alpha_{2}}-1\right)}{\beta_{12} \beta_{21}-\mathrm{K}_{1} \mathrm{~K}_{2}}, \\
& \overline{\mathrm{N}}_{2}=\frac{\beta_{21}\left(\frac{1}{\alpha_{2}}-1\right)-\mathrm{K}_{1}\left(\frac{1}{\alpha_{2}}-1\right)}{\beta_{12} \beta_{21}-\mathrm{K}_{2} \mathrm{~K}_{1}}, \overline{\mathrm{N}}_{3}=0
\end{aligned}
$$

Co- existent state or the Normal steady state:

$\mathrm{E}_{8}$ :

$$
\begin{aligned}
& \bar{N}_{1}=\frac{\mathrm{K}_{2}\left(1-\frac{1}{\alpha_{1}}\right)-\beta_{12}\left(1-\frac{1}{\alpha_{2}}\right)+\frac{1}{\mathrm{~K}_{3}}\left(\beta_{12} \gamma_{23}+\gamma_{13} \mathrm{~K}_{2}\right)\left(1-\frac{1}{\alpha_{3}}\right)}{\mathrm{K}_{1} \mathrm{~K}_{2}+\beta_{12} \beta_{21}}, \\
& \bar{N}_{2}=\frac{\beta_{21}\left(1-\frac{1}{\alpha_{1}}\right)+\mathrm{K}_{1}\left(1-\frac{1}{\alpha_{2}}\right)-\frac{1}{\mathrm{~K}_{3}}\left(\mathrm{~K}_{1} \gamma_{23}-\gamma_{13} \beta_{21}\right)\left(1-\frac{1}{\alpha_{3}}\right)}{\mathrm{K}_{1} \mathrm{~K}_{2}+\beta_{12} \beta_{21}}
\end{aligned}
$$

$$
\overline{\mathrm{N}}_{3}=0
$$




\section{Asymptotic Stability of the Equilibrium States}

Consider small perturbations $\mathrm{v}_{\mathrm{i}}(\mathrm{t}), \mathrm{i}=1,2,3$ over the equilibrium state. The perturbations are so small that their squares and products are neglected.

That is,

$$
\mathrm{N}_{1}=\overline{\mathrm{N}}_{1}+\mathrm{v}_{1}(\mathrm{t}), \quad \mathrm{N}_{2}=\overline{\mathrm{N}}_{2}+\mathrm{v}_{2}(\mathrm{t}), \mathrm{N}_{3}=\overline{\mathrm{N}}_{3}+\mathrm{v}_{3}(\mathrm{t})
$$

Substituting in the difference equations (3.2) we have the equations of perturbations

$$
\begin{aligned}
& \left.\mathrm{v}_{1}(\mathrm{t}+1) \approx \alpha_{1} \overline{\mathrm{N}}_{1}+\alpha_{1} \mathrm{v}_{1}-\mathrm{a}_{11} \overline{\mathrm{N}}_{1}^{2}-2 \mathrm{a}_{11} \overline{\mathrm{N}}_{1} \mathrm{v}_{1}-\mathrm{a}_{12} \overline{\mathrm{N}}_{1} \overline{\mathrm{N}}_{2}-\mathrm{a}_{12} \overline{\mathrm{N}}_{1} \mathrm{v}_{2}-\mathrm{a}_{12} \overline{\mathrm{N}}_{1} \mathrm{v}_{1}+\mathrm{a}_{13} \overline{\mathrm{N}} 1 \overline{\mathrm{N}} 3+\mathrm{a}_{13} \overline{\mathrm{N}} 1 \mathrm{v}_{3}\right) \\
& +\mathrm{a}_{13} \overline{\mathrm{N}} 3 \mathrm{v}_{1}-\overline{\mathrm{N}}_{1} \\
& \mathrm{v}_{2}(\mathrm{t}+1) \approx \alpha_{2} \overline{\mathrm{N}}_{2}+\alpha_{2} \mathrm{v}_{2}-\mathrm{a}_{22} \overline{\mathrm{N}}_{2}^{2}-2 \mathrm{a}_{22} \overline{\mathrm{N}}_{2} \mathrm{v}_{2}-\mathrm{a}_{21} \overline{\mathrm{N}}_{1} \overline{\mathrm{N}}_{2}+\mathrm{a}_{21} \overline{\mathrm{N}}_{2} \mathrm{v}_{1}+\mathrm{a}_{21} \overline{\mathrm{N}}_{1} \mathrm{v}_{2}-\mathrm{a}_{23} \overline{\mathrm{N}}_{2} \overline{\mathrm{N}}_{3}-\mathrm{a}_{23} \mathrm{v}_{3} \overline{\mathrm{N}}_{2} \\
& -\mathrm{a}_{23} \mathrm{v}_{2} \overline{\mathrm{N}}_{3}-\overline{\mathrm{N}}_{2} \\
& \mathrm{v}_{3}(\mathrm{t}+1) \approx \alpha_{3} \overline{\mathrm{N}}_{3}+\alpha_{3} \mathrm{v}_{3}-\mathrm{a}_{33} \overline{\mathrm{N}}_{3}^{2}-2 \mathrm{a}_{33} \overline{\mathrm{N}}_{3} \mathrm{v}_{3}-\overline{\mathrm{N}}_{3}
\end{aligned}
$$

5.1. $\overline{\mathrm{N}}_{1}(\mathrm{t})=0, \quad \overline{\mathrm{N}}_{2}(\mathrm{t})=0, \overline{\mathrm{N}}_{3}(\mathrm{t})=0$

Substituting in the perturbation equations (5.1) and neglecting squares and products, we have the perturbation equations for the present state

$$
\begin{aligned}
& \left.\left.\begin{array}{rl}
\mathrm{v}_{1}(\mathrm{t}+1)=\alpha_{1} \mathrm{v}_{1}\left[1-\mathrm{K}_{1} \mathrm{v}_{1}-\beta_{12} \mathrm{v}_{2}+\gamma_{13} \mathrm{v}_{3}\right] \approx \alpha_{1} \mathrm{v}_{1}(\mathrm{t}) \\
\mathrm{v}_{2}(\mathrm{t}+1)=\alpha_{2} \mathrm{v}_{2}\left[1-\mathrm{K}_{2} \mathrm{v}_{2}-\beta_{21} \mathrm{v}_{1}\right.
\end{array}\right\}+\gamma_{23} \mathrm{v}_{3}\right] \approx \alpha_{2} \mathrm{v}_{2}(\mathrm{t}) \\
& \mathrm{v}_{3}(\mathrm{t}+1)=\alpha_{3} \mathrm{v}_{3}\left[1-\mathrm{K}_{3} \mathrm{v}_{3}\right] \approx \alpha_{3} \mathrm{v}_{3}(\mathrm{t}) \\
& {\left[\begin{array}{ccc}
\alpha_{1} & 0 & 0 \\
0 & \alpha_{2} & 0 \\
0 & 0 & \alpha_{3}
\end{array}\right]}
\end{aligned}
$$

And the Eigen values are $\lambda_{1}=\alpha_{1}, \lambda_{2}=\alpha_{2}$ and $\lambda_{3}=\alpha_{3}$ which are real and positive. For stability analysis, we can consider two cases:

Case 5.1.1: $a_{1}>0, a_{2}>0$ and $a_{3}>0$

here, clearly $\left|\lambda_{\mathrm{i}}\right|>1, \mathrm{i}=1,2,3$.

That is, all the Eigen values lie outside the unit circle. Therefore, the Equilibrium point or fixed point is hyperbolic and is unstable. The asymptotic nature of the three variables $\mathrm{v}_{1}, \mathrm{v}_{2}, \mathrm{v}_{3}$ and the surface plot of the trajectories of the perturbed equations when $a_{1}, a_{2}, a_{3}>0$ are respectively shown in Fig. 2 and Fig. 3.

Case 5.1.2: $a_{1}=0, a_{2}=0$ and $a_{3}=0$

In this case, we have $\left|\lambda_{i}\right|=1, i=1,2,3$.

which means the present state is Spectrally stable or Linearly stable which is shown in Figure 4 and Figure 5. 


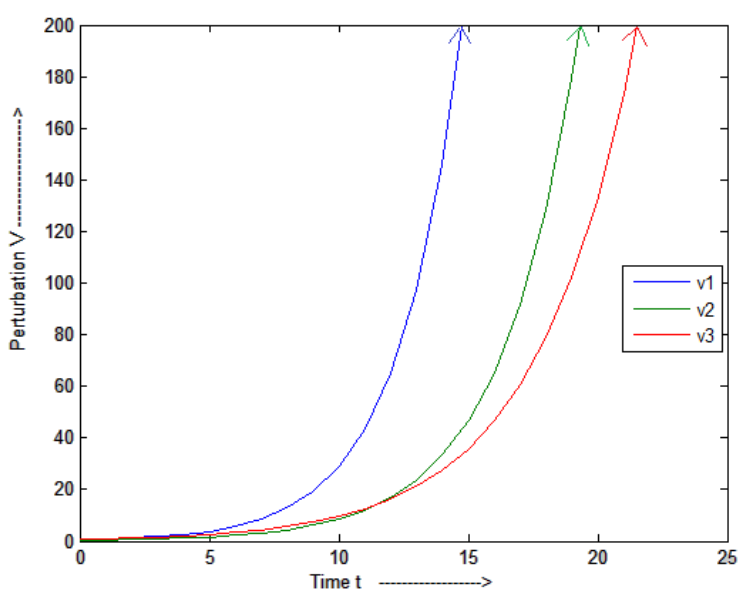

Figure 2. $\mathrm{a}_{1}>0, \mathrm{a}_{2}>0$, and $\mathrm{a}_{3}>0$

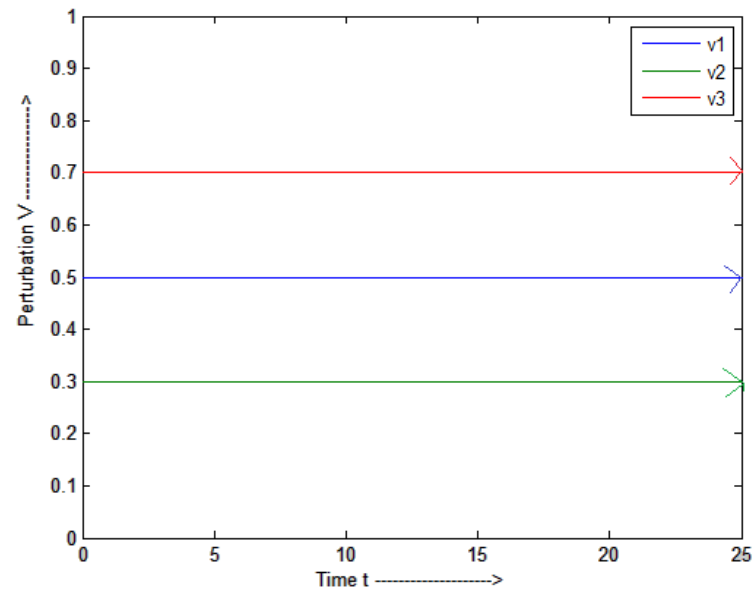

Figure 4. $\mathrm{a}_{1}=0, \mathrm{a}_{2}=0$, and $\mathrm{a}_{3}=0$

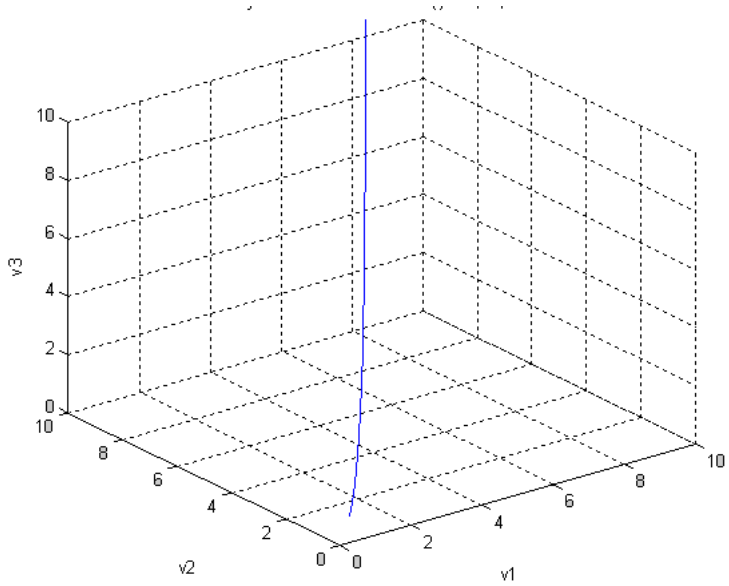

Figure 3. $\mathrm{a}_{1}>0, \mathrm{a}_{2}>0$, and $\mathrm{a}_{3}>0$

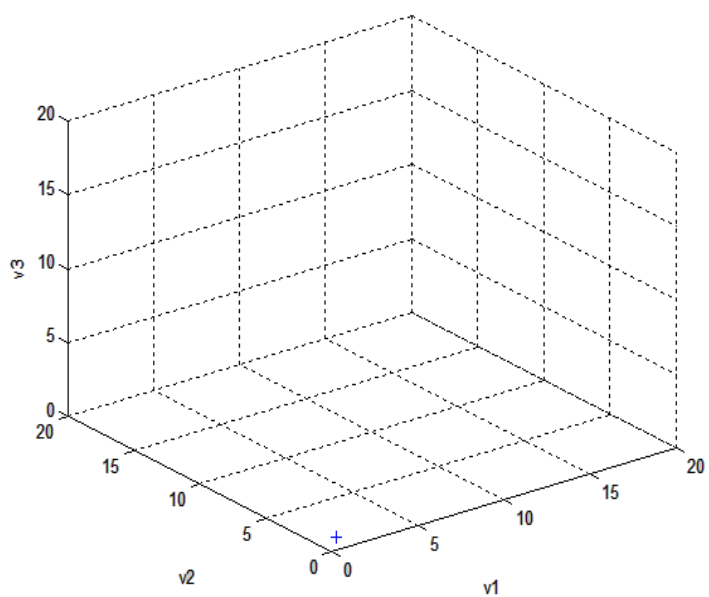

Figure 5. $a_{1}=0, a_{2}=0$, and $a_{3}=0$

B. States in which two species are washed out:

\subsection{The State in which $S_{1}$ and $S_{2}$ Washed out While $S_{3}$ is Not}

$$
\overline{\mathrm{N}}_{1}(\mathrm{t})=0, \quad \overline{\mathrm{N}}_{2}(\mathrm{t})=0, \quad \overline{\mathrm{N}}_{3}(\mathrm{t})=\frac{1}{\mathrm{~K}_{3}}\left(1-\frac{1}{\alpha_{3}}\right)
$$

Substitution in the perturbation equations (5.1) result in

$$
\begin{aligned}
& \mathrm{v}_{1}(\mathrm{t}+1)=\frac{\mathrm{a}_{33} \alpha_{1}+\mathrm{a}_{13}\left(\alpha_{3}-1\right)}{\mathrm{a}_{33}} \mathrm{v}_{1}(\mathrm{t}) \\
& \mathrm{v}_{2}(\mathrm{t}+1)=\frac{\mathrm{a}_{33} \alpha_{2}-\mathrm{a}_{23}\left(\alpha_{3}-1\right)}{\mathrm{a}_{33}} \mathrm{v}_{2}(\mathrm{t}) \\
& \mathrm{v}_{3}(\mathrm{t}+1)=\left(2-\alpha_{3}\right) \mathrm{v}_{3}(\mathrm{t})
\end{aligned}
$$

From the equations (5.2.1) the Jacobian matrix is 


$$
\left[\begin{array}{ccc}
\frac{\mathrm{a}_{33} \alpha_{1}+\mathrm{a}_{13}\left(\alpha_{3}-1\right)}{\mathrm{a}_{33}} & 0 & 0 \\
0 & \frac{\mathrm{a}_{33} \alpha_{2}-\mathrm{a}_{23}\left(\alpha_{3}-1\right)}{\mathrm{a}_{33}} & 0 \\
0 & 0 & \left(2-\alpha_{3}\right)
\end{array}\right]
$$

And the Eigen values are $\lambda=\frac{\mathrm{a}_{33} \alpha_{1}+\mathrm{a}_{13}\left(\alpha_{3}-1\right)}{\mathrm{a}_{33}}, \frac{\mathrm{a}_{33} \alpha_{2}-\mathrm{a}_{23}\left(\alpha_{3}-1\right)}{\mathrm{a}_{33}}$ and $2-\alpha_{3}$.

\section{Stability analysis}

For this state to be stable, all the Eigen values must be inside the unit circle. That is,

$$
\begin{aligned}
& \left|\lambda_{1}\right|<1 \Leftrightarrow 1<-a_{33}\left(2+a_{1}\right)<a_{13}\left(\alpha_{3}-1\right) \\
& <a_{33}\left(1-\alpha_{1}\right) \Leftrightarrow-a_{33}\left(2+a_{1}\right)<a_{13} a_{3}<-a_{33} a_{1} \\
& \left|\lambda_{2}\right|<1 \Leftrightarrow a_{33} a_{2}<a_{23} a_{3}<a_{33}\left(2+a_{2}\right) \\
& \left|\lambda_{3}\right|<1 \Leftrightarrow 1<\alpha_{3}<3 \Leftrightarrow 0<a_{3}<2
\end{aligned}
$$

Of the above in (5.2.2.), in the first inequality, the quantity in the middle i.e. $\mathrm{a}_{13} \mathrm{a}_{3}$ is positive. Therefore, the condition is invalid. Hence this state is unstable. This is diagrammatically shown in Figure 6 and Figure 7.

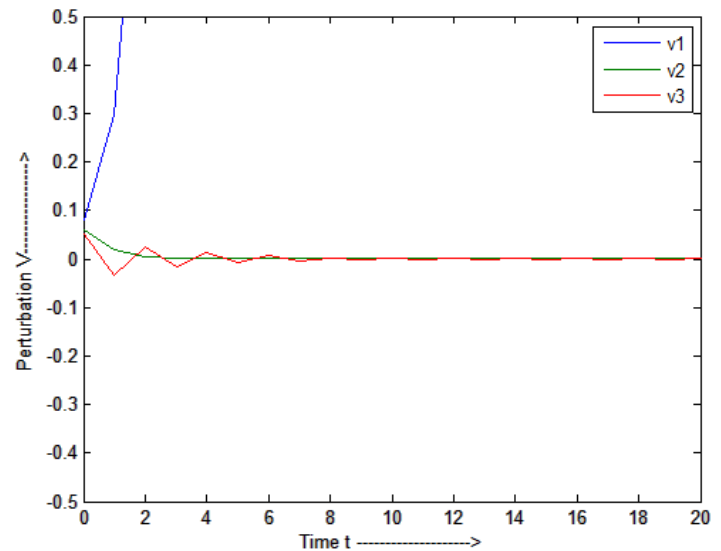

Figure 6. $\mathrm{S}_{1}$ and $\mathrm{S}_{2}$ washed out state

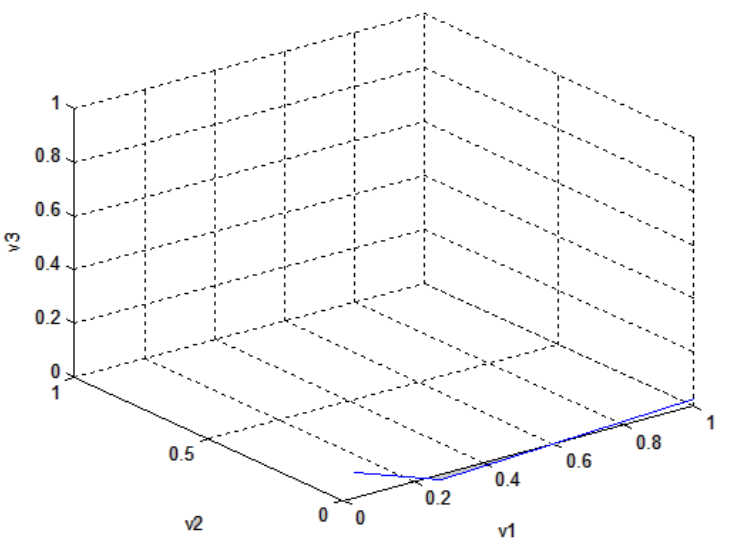

Figure 7. $\mathrm{S}_{1}$ and $\mathrm{S}_{2}$ washed out state

\subsection{The State in which $S_{1}$ and $S_{3}$ Washed out But $S_{2}$ is Not}

$$
\overline{\mathrm{N}}_{1}=0, \quad \overline{\mathrm{N}}_{2}=\frac{1}{\mathrm{~K}_{2}}\left(1-\frac{1}{\alpha_{2}}\right), \overline{\mathrm{N}}_{3}=0
$$

The perturbation equations for the present state from (5.1) are

$$
\left.\begin{array}{l}
\mathrm{v}_{1}(\mathrm{t}+1)=\left[\alpha_{1}-\frac{\mathrm{a}_{12}}{\mathrm{a}_{22}}\left(\alpha_{2}-1\right)\right] \mathrm{v}_{1}(\mathrm{t}) \\
\mathrm{v}_{2}(\mathrm{t}+1)=\frac{\mathrm{a}_{21}}{\mathrm{a}_{22}}\left(\alpha_{2}-1\right) \mathrm{v}_{2}(\mathrm{t})+\left(2-\alpha_{2}\right) \mathrm{v}_{2}(\mathrm{t})-\frac{\mathrm{a}_{23}}{\mathrm{a}_{22}}\left(\alpha_{2}-1\right) \mathrm{v}_{3}(\mathrm{t}) \\
\mathrm{v}_{3}(\mathrm{t}+1)=\alpha_{3} \mathrm{v}_{3}(\mathrm{t})
\end{array}\right\}
$$


From the equations (5.3.1) the Jacobian matrix is

$$
\left[\begin{array}{ccc}
\alpha_{1}-\frac{\mathrm{a}_{12}}{\mathrm{a}_{22}}\left(\alpha_{2}-1\right) & 0 & 0 \\
\frac{\mathrm{a}_{21}}{\mathrm{a}_{22}}\left(\alpha_{2}-1\right) & 2-\alpha_{2} & -\frac{\mathrm{a}_{23}}{\mathrm{a}_{22}}\left(\alpha_{2}-1\right) \\
0 & 0 & \alpha_{3}
\end{array}\right]
$$

And the Eigen values are $\lambda_{1}=\alpha_{1}-\frac{a_{12}}{a_{22}}\left(\alpha_{2}-1\right), \lambda_{2}=2-\alpha_{2}$ and $\lambda_{3}=\alpha_{3}$

\section{Stability analysis}

The conditions for stability of the equilibrium state are

$$
\left.\begin{array}{l}
\left|\lambda_{1}\right|<1 \Leftrightarrow 1<a_{1} a_{22}<a_{2} a_{12}<\left(2+a_{1}\right) a_{22} \\
\left|\lambda_{2}\right|<1 \Leftrightarrow 1<\alpha_{2}<3 \\
\left|\lambda_{3}\right|=1 \Leftrightarrow a_{3}=0
\end{array}\right\}
$$

In (5.3.2) from the first two inequalities it is clear that for a particular set of values of the parameters, the first two Eigen values lie inside the unit circle. From the third inequality we can conclude that when $\mathrm{a}_{3}=0$, the Eigen value $\lambda_{3}$ lies on the unit circle. Therefore this equilibrium state is Linearly or spectrally stable. The results are shown in Figure 8 and Figure 9.

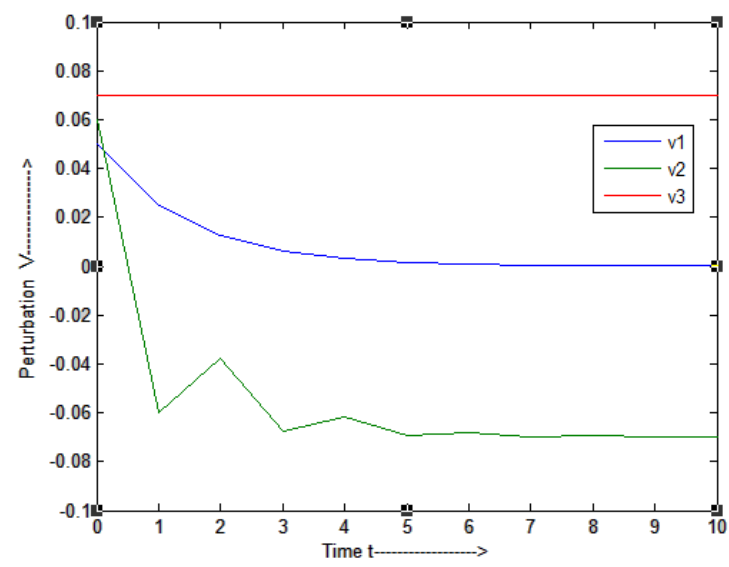

Figure 8. S1 and S3 washed out state

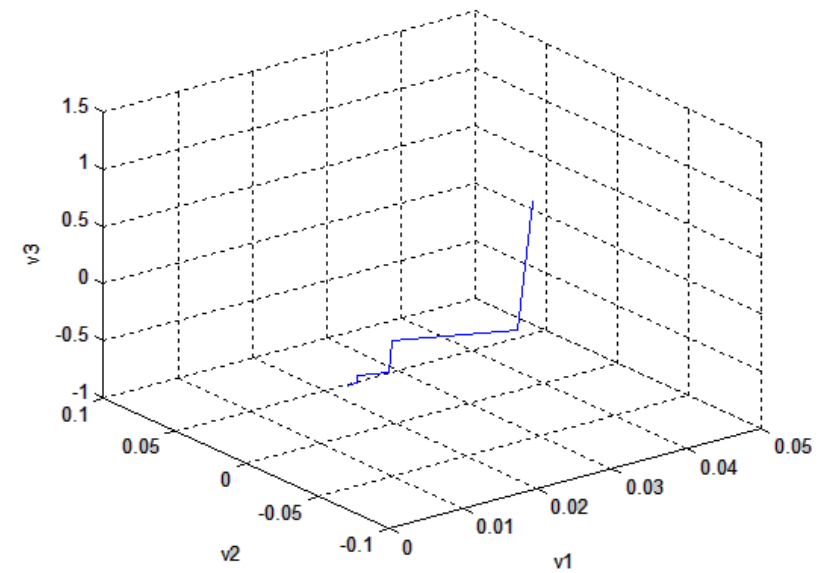

Figure 9. $\mathrm{S}_{1}$ and $\mathrm{S}_{3}$ washed out state

C. States in which only one species is washed out

\subsection{The State in which the Species $S_{1}$ is Washed out while $S_{2}$ and $S_{3}$ are Not}

$$
\begin{aligned}
& \overline{\mathrm{N}}_{1}=0, \\
& \overline{\mathrm{N}}_{2}=\frac{1}{\mathrm{~K}_{2}}\left\{1-\frac{1}{\alpha_{2}}-\frac{\gamma_{23}}{\mathrm{~K}_{3}}\left(1-\frac{1}{\alpha_{2}}\right)\right\} \\
& \overline{\mathrm{N}}_{3}=\frac{1}{\mathrm{~K}_{3}}\left(1-\frac{1}{\alpha_{3}}\right) .
\end{aligned}
$$

Substituting in the perturbation equations (5.1) and neglecting squares and products, we have the perturbation equations for the present state 


$$
\begin{aligned}
& \mathrm{v}_{1}(\mathrm{t}+1)=\left(\alpha_{1}-\frac{\mathrm{a}_{12}\left(\mathrm{a}_{2} \mathrm{a}_{33}-\mathrm{a}_{3} \mathrm{a}_{23}\right)}{\mathrm{a}_{22} \mathrm{a}_{33}}+\frac{\mathrm{a}_{1} \mathrm{a}_{13}}{\mathrm{a}_{33}}\right) \mathrm{v}_{1}(\mathrm{t}) \\
& \mathrm{v}_{2}(\mathrm{t}+1)=\mathrm{a}_{21} \frac{\mathrm{a}_{23}\left(1-\alpha_{3}\right)+\mathrm{a}_{33}\left(\alpha_{2}-1\right)}{\mathrm{a}_{22} \mathrm{a}_{33}} \mathrm{v}_{1}(\mathrm{t})+\left(1-\mathrm{a}_{2}+\frac{\mathrm{a}_{3} \mathrm{a}_{23}}{\mathrm{a}_{33}}\right) \mathrm{v}_{2}(\mathrm{t})+\frac{\mathrm{a}_{23}\left(\mathrm{a}_{3} \mathrm{a}_{23}-\mathrm{a}_{2} \mathrm{a}_{33}\right)}{\mathrm{a}_{22} \mathrm{a}_{33}} \mathrm{v}_{3}(\mathrm{t}) \\
& +\alpha_{2} \frac{\mathrm{a}_{23}\left(1-\alpha_{3}\right)+\mathrm{a}_{33}\left(\alpha_{2}-1\right)}{\mathrm{a}_{22} \mathrm{a}_{33}}-\mathrm{a}_{22}\left(\frac{\mathrm{a}_{23}\left(1-\alpha_{3}\right)+\mathrm{a}_{33}\left(\alpha_{2}-1\right)^{2}}{\mathrm{a}_{22} \mathrm{a}_{33}}\right) \\
& -\alpha_{2} \gamma_{23} \frac{\mathrm{a}_{23}\left(1-\alpha_{3}\right)+\mathrm{a}_{33}\left(\alpha_{2}-1\right)}{\mathrm{a}_{22} \mathrm{a}_{33}} \frac{1}{\mathrm{~K}_{3}} \frac{\alpha_{3}-1}{\alpha_{3}}-\frac{\mathrm{a}_{23}\left(1-\alpha_{3}\right)+\mathrm{a}_{33}\left(\alpha_{2}-1\right)}{\mathrm{a}_{22} \mathrm{a}_{33}} \\
& \mathrm{v}_{3}(\mathrm{t}+1)=\left(2-\alpha_{3}\right) \mathrm{v}_{3}(\mathrm{t})
\end{aligned}
$$

From the above equations the Jacobian matrix is

$$
\left[\begin{array}{ccc}
a_{1}-\frac{a_{12}\left(a_{2} a_{33}-a_{3} a_{23}\right)}{a_{22} a_{33}}+\frac{a_{1} a_{13}}{a_{33}} & 0 & 0 \\
a_{21} \frac{a_{23}\left(1-a_{3}\right)+a_{33}\left(a_{2}-1\right)}{a_{22} a_{33}} & 1-a_{2}+\frac{a_{3} a_{23}}{a_{33}} & \frac{a_{23}\left(a_{3} a_{23}-a_{2} a_{33}\right)}{a_{22} a_{33}} \\
0 & 0 & 2-a_{3}
\end{array}\right]
$$

And the Eigen values are

$$
\lambda_{1}=\alpha_{1}-\frac{a_{12}\left(a_{2} a_{33}-a_{3} a_{23}\right)}{a_{22} a_{33}}+\frac{a_{1} a_{13}}{a_{33}}, \lambda_{2}=1-a_{2}+\frac{a_{3} a_{23}}{a_{33}} \text { and } \lambda_{3}=2-\alpha_{3}
$$

Examining the conditions for stability of this equilibrium state, we have

$$
\left.\begin{array}{l}
\left|\lambda_{3}\right|<1 \Leftrightarrow 1<\alpha_{3}<3 \\
\left|\lambda_{2}\right|<1 \Leftrightarrow 1-a_{2}+\frac{a_{3} a_{23}}{a_{33}}<1 \\
\left|\lambda_{1}\right|<1 \Leftrightarrow \alpha_{1}-\frac{a_{12}\left(a_{2} a_{33}-a_{3} a_{23}\right)}{a_{22} a_{33}}
\end{array}\right\}<1
$$

The inequalities (5.4.2) are not valid because $\mathrm{a}_{2} \mathrm{a}_{33}-\mathrm{a}_{3} \mathrm{a}_{23}>0$. Therefore, the present state is unstable. The results for a specific case are shown in Figures 10 and Figure 11.

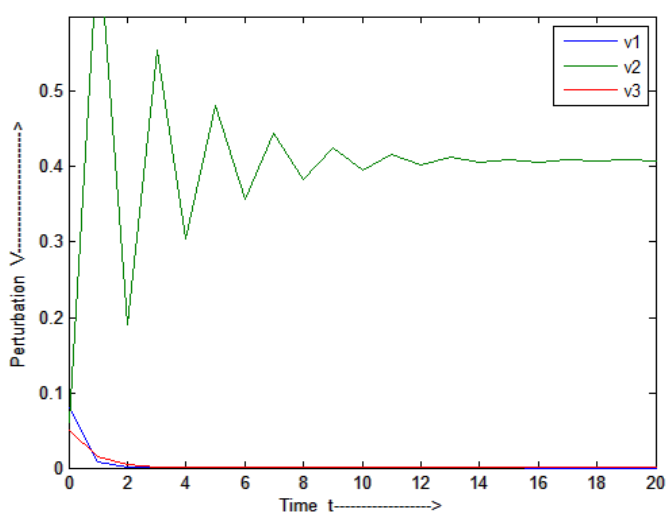

Figure 10. $S_{1}$ washed out state

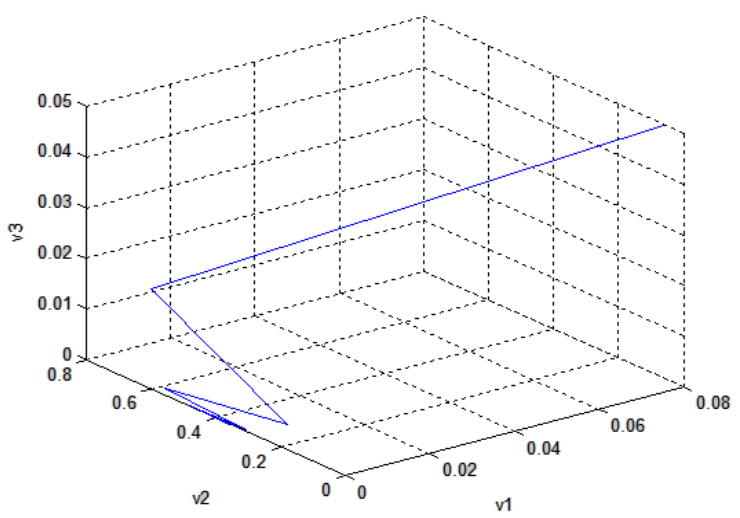

Figure 11. $\mathrm{s}_{1}$ washed out state 


\subsection{State in Which the Species $S_{2}$ is Washed Out While $S_{1}$ and $S_{3}$ are Not}

$$
\begin{aligned}
& \overline{\mathrm{N}}_{1}=\frac{1}{\mathrm{~K}_{1}}\left\{1-\frac{1}{\alpha_{2}}-\frac{\gamma_{13}}{\mathrm{~K}_{3}}\left(1-\frac{1}{\alpha_{3}}\right)\right\}>0 \\
& \text { (i.e. } \left.\mathrm{a}_{1} \mathrm{a}_{33}+\mathrm{a}_{3} \mathrm{a}_{13}>0\right), \\
& \overline{\mathrm{N}}_{2}=0 \text { and } \\
& \overline{\mathrm{N}}_{3}=\frac{1}{\mathrm{~K}_{3}}\left(1-\frac{1}{\alpha_{3}}\right)
\end{aligned}
$$

The perturbation equations for the present state resulting from (5.1) by neglecting squares and products are

$$
\left.\begin{array}{rl}
\mathrm{v}_{1}(\mathrm{t}+1)= & \left.\alpha_{1}\left(1+\gamma_{13} \overline{\mathrm{N}}_{3}-2 \mathrm{~K}_{1} \overline{\mathrm{N}}_{1}\right) \mathrm{v}_{1}(\mathrm{t})-\alpha_{1} \beta_{12} \overline{\mathrm{N}}_{1} \mathrm{v}_{2}(\mathrm{t})+\alpha_{1} \gamma_{13} \overline{\mathrm{N}}_{1} \mathrm{v}_{3}(\mathrm{t})+\alpha_{1} \overline{\mathrm{N}}_{1}-\alpha_{1} \mathrm{~K}_{1} \overline{\mathrm{N}}_{1}^{2}+\alpha_{1} \gamma_{13} \overline{\mathrm{N}}_{1} \overline{\mathrm{N}}_{3}-\overline{\mathrm{N}}_{1}\right) \\
= & \left(1-\mathrm{a}_{1}-\frac{\mathrm{a}_{3} \mathrm{a}_{13}}{\mathrm{a}_{33}}\right) \mathrm{v}_{1}(\mathrm{t})-\frac{\mathrm{a}_{12}}{\mathrm{a}_{11}}\left(\mathrm{a}_{1}+\frac{\mathrm{a}_{3} \mathrm{a}_{13}}{\mathrm{a}_{33}}\right) \mathrm{v}_{2}(\mathrm{t})+\frac{\mathrm{a}_{13}}{\mathrm{a}_{11}}\left(\mathrm{a}_{1}+\frac{\mathrm{a}_{3} \mathrm{a}_{13}}{\mathrm{a}_{33}}\right) \mathrm{v}_{3}(\mathrm{t})+\alpha_{1} \overline{\mathrm{N}}_{1}-\alpha_{1} \mathrm{~K}_{1} \overline{\mathrm{N}}_{1}^{2} \\
& +\alpha_{1} \gamma_{13} \overline{\mathrm{N}}_{1} \overline{\mathrm{N}}_{3}-\overline{\mathrm{N}}_{1} \\
\mathrm{v}_{2}(\mathrm{t}+1)= & \alpha_{2}\left(1+\beta_{21} \overline{\mathrm{N}}_{1}-\gamma_{23} \overline{\mathrm{N}}_{3}\right) \mathrm{v}_{2}(\mathrm{t}) \\
= & \left(\alpha_{2}+\frac{\mathrm{a}_{21}}{\mathrm{a}_{11}}\left(\mathrm{a}_{1}+\frac{\mathrm{a}_{3} \mathrm{a}_{13}}{\mathrm{a}_{33}}\right)-\frac{\mathrm{a}_{3} \mathrm{a}_{23}}{\mathrm{a}_{33}}\right) \mathrm{v}_{2}(\mathrm{t}) \\
\mathrm{v}_{3}(\mathrm{t}+1)= & \alpha_{3}\left(1-2 \mathrm{~K}_{3} \overline{\mathrm{N}}_{3}\right) \mathrm{v}_{3}(\mathrm{t})+\alpha_{3} \overline{\mathrm{N}}_{3}-\alpha_{3} \mathrm{~K}_{3} \overline{\mathrm{N}}_{3}^{2}-\overline{\mathrm{N}}_{3} \\
= & \left(2-\alpha_{3}\right) \mathrm{v}_{3}(\mathrm{t})+\alpha_{3} \overline{\mathrm{N}}_{3}-\alpha_{3} \mathrm{~K}_{3} \overline{\mathrm{N}}_{3}^{2}-\overline{\mathrm{N}}_{3}
\end{array}\right\}
$$

From the above equations the Jacobian matrix is

$$
\left[\begin{array}{ccc}
1-a_{1}-\frac{a_{3} a_{13}}{a_{33}} & -\frac{a_{12}}{a_{11}}\left(a_{1}+\frac{a_{3} a_{13}}{a_{33}}\right) & \frac{a_{13}}{a_{11}}\left(a_{1}+\frac{a_{3} a_{13}}{a_{33}}\right) \\
0 & a_{2}+\frac{a_{21}}{a_{11}}\left(a_{1}+\frac{a_{3} a_{13}}{a_{33}}\right)-\frac{a_{3} a_{23}}{a_{33}} & 0 \\
0 & 0 & 2-\alpha_{3}
\end{array}\right]
$$

And the Eigen values are

$$
\begin{aligned}
& \lambda_{1}=1-a_{1}-\frac{a_{3} a_{13}}{a_{33}} \\
& \lambda_{2}=\alpha_{2}+\frac{a_{21}}{a_{11}}\left(a_{1}+\frac{a_{3} a_{13}}{a_{33}}\right)-\frac{a_{3} a_{23}}{a_{33}} \\
& \lambda_{3}=2-\alpha_{3}
\end{aligned}
$$

Examining the conditions for stability of this equilibrium state, we have

$$
\begin{aligned}
& \left|\lambda_{1}\right|<1 \Leftrightarrow a_{3} a_{13}<\left(2-a_{1}\right) a_{33} \text { and } a_{1}>2 \\
& \left|\lambda_{2}\right|<1 \Leftrightarrow a_{11}\left(a_{3} a_{23}-\left(2+a_{2}\right) a_{33}\right)<a_{21}\left(a_{1} a_{33}+a_{3} a_{13}\right)<a_{11}\left(a_{3} a_{23}-a_{2} a_{33}\right) \\
& \text { and } a_{3} a_{23}>a_{2} a_{33} \\
& \left|\lambda_{3}\right|<1 \Leftrightarrow 1<\alpha_{3}<3
\end{aligned}
$$

Since all the conditions (inequalities (5.5.2)) above are feasible, for the above conditions the Ligen values lie inside a unit circle. So the equilibrium state is stable.

The results are shown in Figure 12 and Figure 13. 


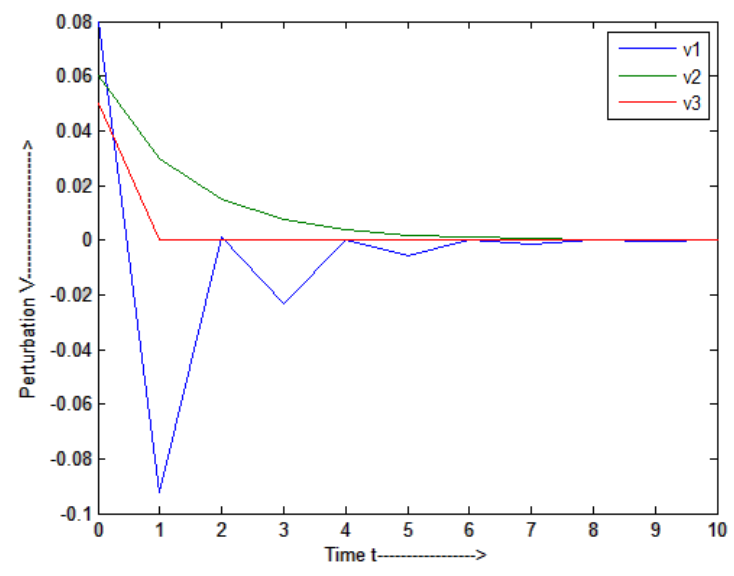

Figure 12. $\mathrm{S}_{2}$ washed out state

\section{Conclusion}

In this paper a three species eco system with various interactions between the species is considered and the asymptotic stability of some of the Equilibrium states is examined. It is observed that among the states, the state in which the Prey and its Host species are washed out (extinct), is spectrally stable and the state where the Predator/Ammensal species is washed out, is asymptotically stable. From this it can be understood that in the former case, though the prey and host species are extinct, the Predator survives due to non inhibition by its enemy. While in the later case, the Prey species and its host species continue to exist because neither is dependent on the predator species for its survival. The results are illustrated with two dimensional plots as well as surface plots.

\section{REFERENCES}

[1] Lotka A.J., "Elements of physical Biology, William \& Wilking Baltimore, 1925.

[2] Volterra V., Leconssen La Theorie Mathematique De La Leitte Pou Lavie, Gautheir Villara, Paris, 1931.

[3] Freedman, H.I., "Deterministic Mathematical Models in population Ecology Marcel-Decker, New York, 1980.

[4] Kapur J.N., "Mathematical modeling in biology and Medicine",Affiliated east west, 1985.

[5] Kapur J.N., "Mathematical modeling, Wiley Eastern, 1985.

[6] Meyer W.J., "Concepts of Mathematical modeling", Mc. Grawhil, 1985.

[7] Paul Colinvaux, Ecology, John Wiley and Sons Inc., New York, 1986.

[8] Srinivas N.C., "Some Mathematical aspects of modeling in

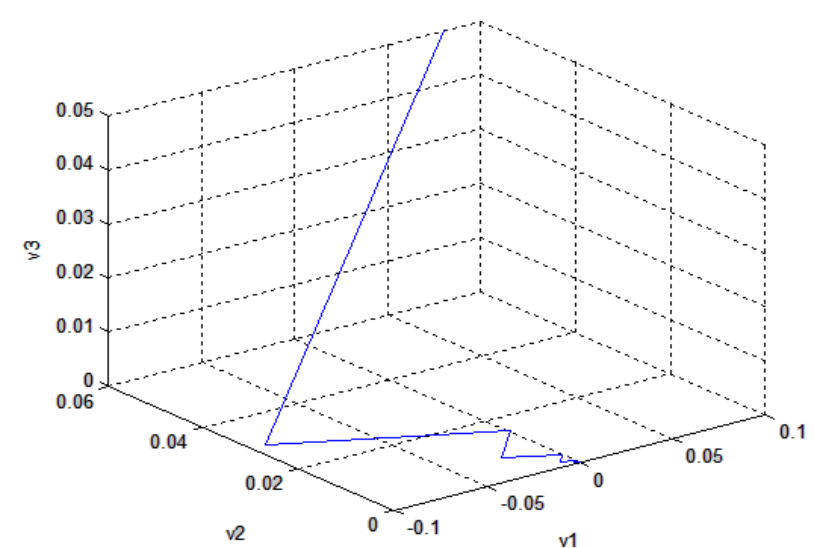

Figure 13. $\mathrm{S}_{2}$ washed out state

Bio-medical sciences". PhD thesis, Kakatiya University, 1991.

[9] Cushing J.M., Integro-Differnetial Equations and Delay Models in Population Dynamics, Lecture Notes in Bio-Mathematics, 20, Springer Verlag, 1997.

[10] Lakshmi Narayana. K., "A mathematical study of a preypredator ecological model with a partial cover for the prey and alternative food for the predator", $\mathrm{PhD}$ thesis, JNTU, 2005.

[11] Lakshmi Narayan.K \& Pattabhi Ramacharyulu N.Ch, 2007, "A prey predator model with cover for prey and alternate food for the predator and time delay", International Journal of Scientific Computing Vol. 1, pg. 7-14.

[12] Ravindra Reddy., "A study on mathematical models of Ecological mutualism between two interacting species" $\mathrm{PhD}$ thesis, O.U, 2008.

[13] Archana Reddy R. Pattabhi Ramacharyulu N. Ch \& Krishna Gandhi. B., "A stability analysis of two competitive interacting species with harvesting of both the species at a constant rate" International Journal of scientific computing (1); pp 57-68, 2007.

[14] Bhaskara Rama Sarma \& Pattabhi Ramacharyulu N.Ch., "Stability analysis of two species competitive ecosystem". International Journal of logic based intelligent systems, Vol.2 No. 1, 2008.

[15] Seshagiri Rao.N \& Pattabhi Ramacharyulu N.Ch., "Stability of a syn-ecosystem consisting of a Prey -Predator and host commensal to the prey-I" (With mortality rate of prey), 2009.

[16] D.Ravi kiran, B.Rami reddy, N.Ch.P.Acharyulu, "A Numerical analysis of stability of a three species Eco system consisting of Prey, Predator and a third species which is host to prey and enemy to Predator" ANU Journal of Physical Sciences, Volume 3, No. 1 \& 2, 2011.

[17] D.Ravi kiran, B.Rami reddy, 'Stability of a three species ecological system consisting of Prey, predator species and a third species which is a host to the prey and enemy to the predator", Journal of Experimental sciences, Vol.3, No.12, 2012. 